\title{
Energetic and exergetic analysis of Rankine cycles for solar power plants with parabolic trough and thermal storage
}

\author{
Victor-Eduard Cenuşă , George Darie, Diana Tuţică, Mihaela Norişor, Florin-Niculae Alexe and \\ Chiriţă-Mihai Muşat \\ Power Engineering Faculty, University Politehnica of Bucharest, Splaiul Independentei, 313, Bucharest 060042, Romania
}

\begin{abstract}
The paper analyzes the "secondary" circuit (for thermodynamic conversion) of a Concentrated Solar Power (CSP) plant with thermodynamic cycle, whose mirrors field supplies a thermal power, averaged over a sunny day, of about $100 \mathrm{MW}$ heat. We study the case of parabolic trough solar collector using silicone oil in the "primary" circuit, which limits the peak temperature below $400{ }^{\circ} \mathrm{C}$. The "primary" circuit uses thermal storage, allowing a delay between the power generation in rapport with the solar energy capture. We choose a water-steam cycle, type Hirn. For increasing its efficiency, it has regenerative feed water preheating and steam reheating. We compared, energetic and exergetic, two types of cycles, using a numerical model with iterative structure, developed by the authors. The results showed that the simplified design achieves practically the same thermodynamic performances with the advanced one.
\end{abstract}

\section{Introduction}

The Concentrated Solar Power (CSP) technology gets to high temperatures, depending of capture system: heliostats and towers [1-3], or parabolic troughs [4-8]. In solar plants with thermodynamic cycle, it allows increasing their efficiency. Additional, use of a heat stock in the "primary" circuit, allows a delay between the solar energy capture and the power generation. The big CSP Plants with Steam Cycle (CSPP-SC) use heliostats and towers with a high concentrating factor, which allow temperatures over $550^{\circ} \mathrm{C}$ and main steam parameters similar to those from classical TPP. In this case, the primary circuit uses molten salts. At medium power, the CSPP-SC uses parabolic trough solar collector and silicone oil. The restrictions given by the concentration factor, and the physical properties of the silicone oil limits the maximum temperature in the primary circuit, while the shape of the heat transfer t-q diagram for Steam Generator (SG) restricts the parameters in the thermodynamic cycle.

The present paper analyzes the "secondary" circuit (for thermodynamic conversion of heat into mechanical work, and power) of a CSPP-SC with parabolic trough, which takes over a heat flow rate, averaged over the duration of a sunny day, of $100 \mathrm{MW}$.

We analyzed both heat transfer processes, as well as thermodynamic energy conversion, separately and in their interconnection, by energetic and exergetic methods.

* e-mail: victor. cenusa@energ.pub.ro

\section{Energetic and exergetic comparative analysis}

The primary circuit diagram is shown in Figure 1.

The first phase of energetic and exergetic analysis was made on the processes of SG. We considered that in the primary circuit the temperatures of the silicon oil are: on the hot branch $688.15 \mathrm{~K}$, and on the cold branch $543.15 \mathrm{~K}$. Taking as reference the cooling water temperature, $295.15 \mathrm{~K}$, we obtained, for a primary heat flow rate of $100 \mathrm{MW}$, an exergy flow rate of 50.071 MW. Further we studied the heat transfer into SG, assigning different parameters in the secondary circuit. The results showed that the steam reheat pressure $\left(p_{\text {reheat }}\right)$ has a low influence over the t-q diagram, and on the exergetic efficiency of heat transfer. The main parameters determining the higher average temperature of the cycle, and the transmitted exergy rate flow are (in order of importance): steam pressure, $p_{0}$, and SG water inlet temperature, $t_{\text {feed water }}$.

The above parameters have opposite effects on the t-q diagram. When $p_{0}$ is rising, $t_{\text {feed water }}$ needs to decrease and when $t_{\text {feed water }}$ increases is necessary to reduce $p_{0}$.

Forwards were analyzed in comparison two types of cycles, both without HP regenerative water preheaters:

- advanced regenerative cycle $\# \# 1$ (Fig. 2). In these one the water preheating is performed in five steps, with $t_{\text {feed water }} \cong 180^{\circ} \mathrm{C}, \quad$ corresponding to $p_{\text {deaerator }} \cong 1.1$ $\mathrm{MPa}$, and the higher steam extraction pressure is at the HPC exhaust $\left(p_{\text {max preheat }}=p_{\text {reheat }}\right)$. The $\mathrm{t}$-q diagram and the exergetic flow restrictions, limit the value of $p_{0}$ at $5.7 \mathrm{MPa}$; 


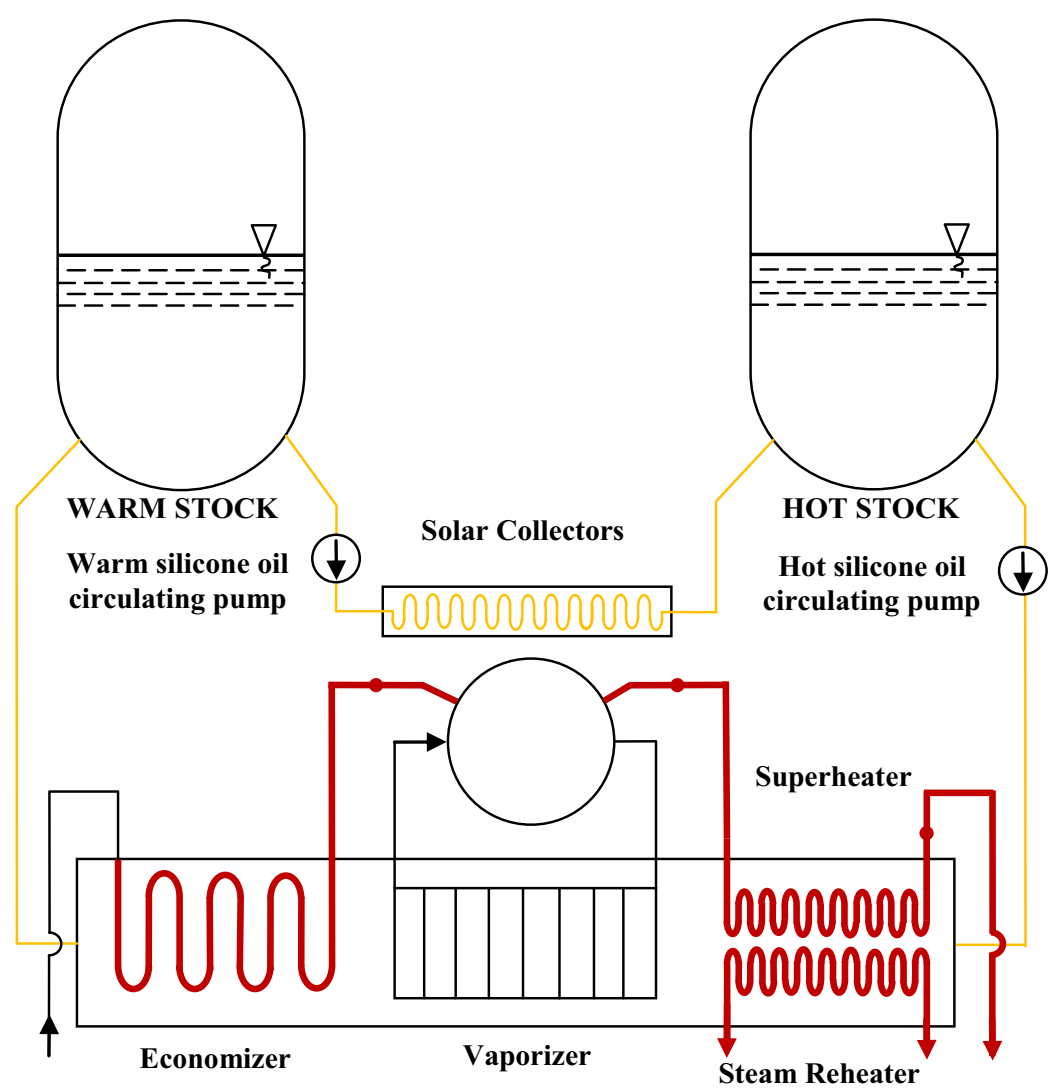

Fig. 1. The diagram of the primary circuit of a CSPP-SC with parabolic trough collectors and thermal storage, including the Steam Generator (SG).

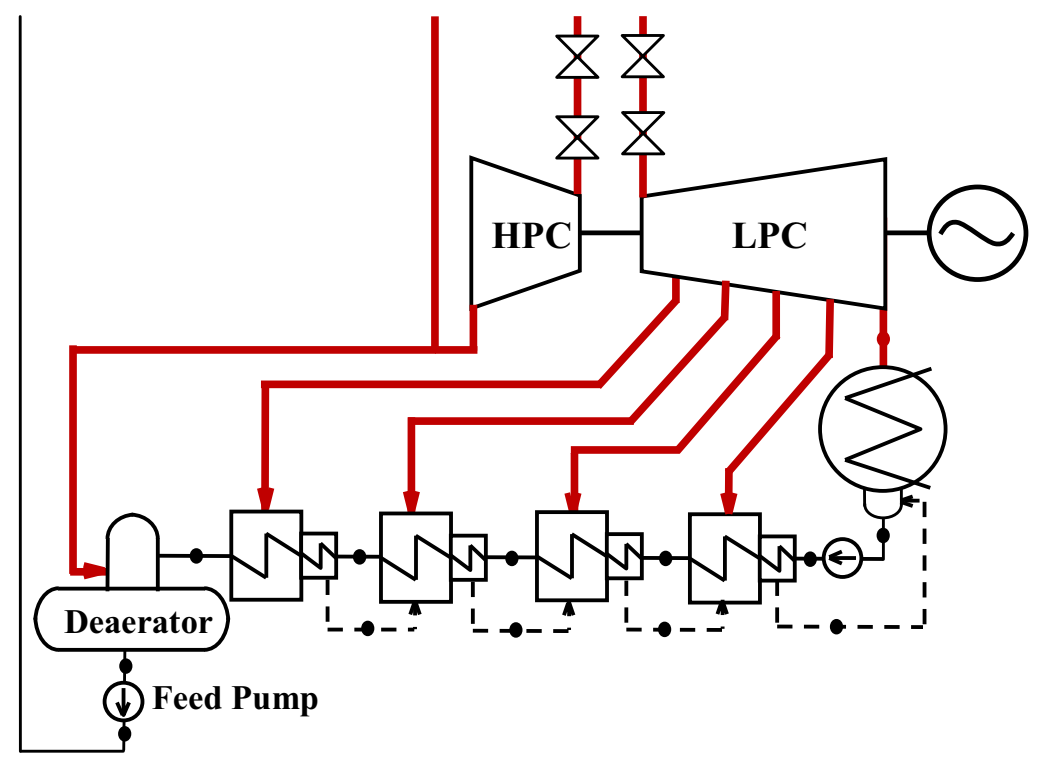

Fig. 2. Advanced regenerative preheating Rankine-Hirn cycle diagram (s\#1). 


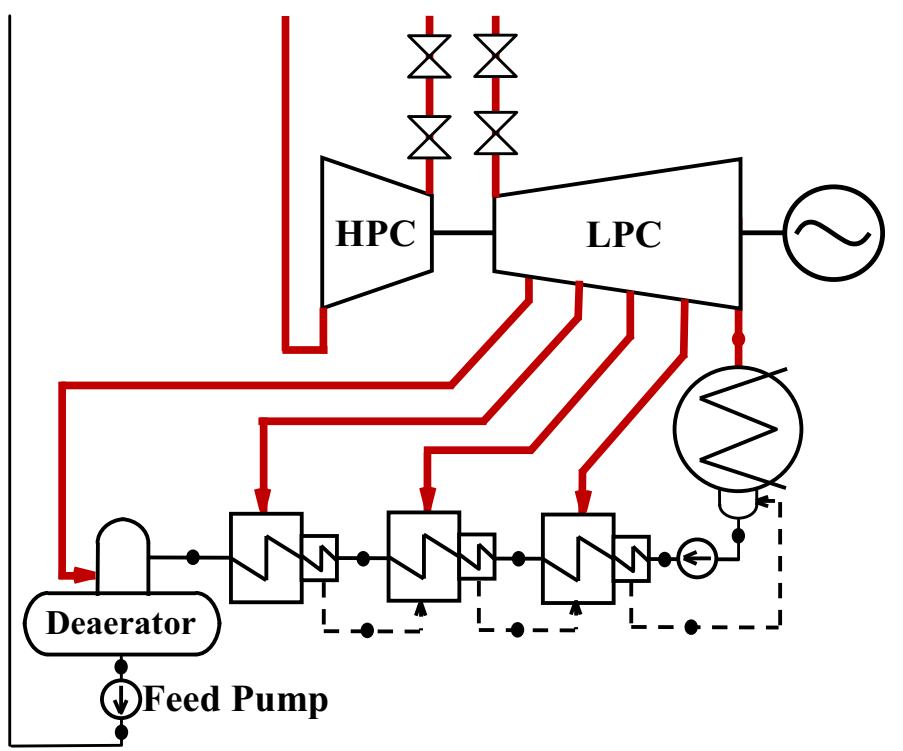

Fig. 3. Limited regenerative preheated Rankine-Hirn cycle diagram (s\#2).

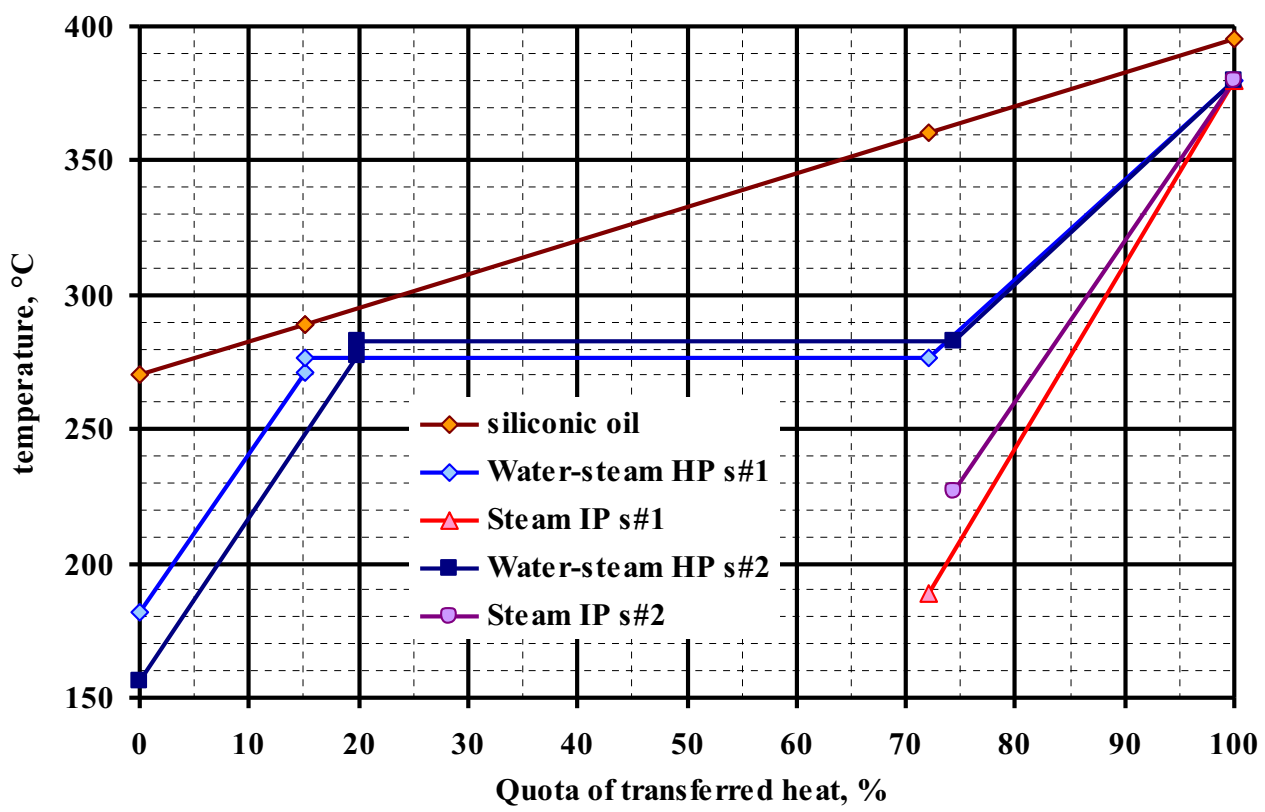

Fig. 4. The t-q heat transfer diagram in SG for the two cases.

- limited regenerative cycles $\# 2$ (Fig. 3), with four preheating stages, all steam extraction in the LPC $\left(p_{\text {max preheat }}<\right.$ $\left.p_{\text {reheat }}\right)$, and $t_{\text {feed water }} \cong 155^{\circ} \mathrm{C}$, corresponding to $p_{\text {deaerator }}$ $\cong 0.55 \mathrm{MPa}$. In this case, the t-q diagram and exergy flow restrictions are more flexible, allowing the increase of $p_{0}$ up to $6.3 \mathrm{MPa}$, and the $p_{\text {reheat }}$ rising too.

Both cases were numerically modeled using software with iterative structure, developed by the authors. Figure 4 enables a comparative analysis of the heat transfer in SG for the two schemes. Mention that in both cases are obtained the same values for:
- the exergetic efficiency of the process in SG: $\eta_{\text {exergetic }}$ $\mathrm{SG} \cong 89.127 \%$;

- the exergy flow rate toward the secondary circuit: $\Phi_{\text {exergetic to water-steam }} \cong 44.628 \mathrm{MW}$.

The second phase in our energetic and exergetic analysis was dedicated to the Steam Cycles (SC) processes, including: the flowing process into pipes and valves, the heat transfer process in water preheaters and condenser, the adiabatic efficiency of the expansion process in Steam Turbines (ST), mechanical losses, generators losses, and others. Figure 5 enables a comparative analysis of the steam 


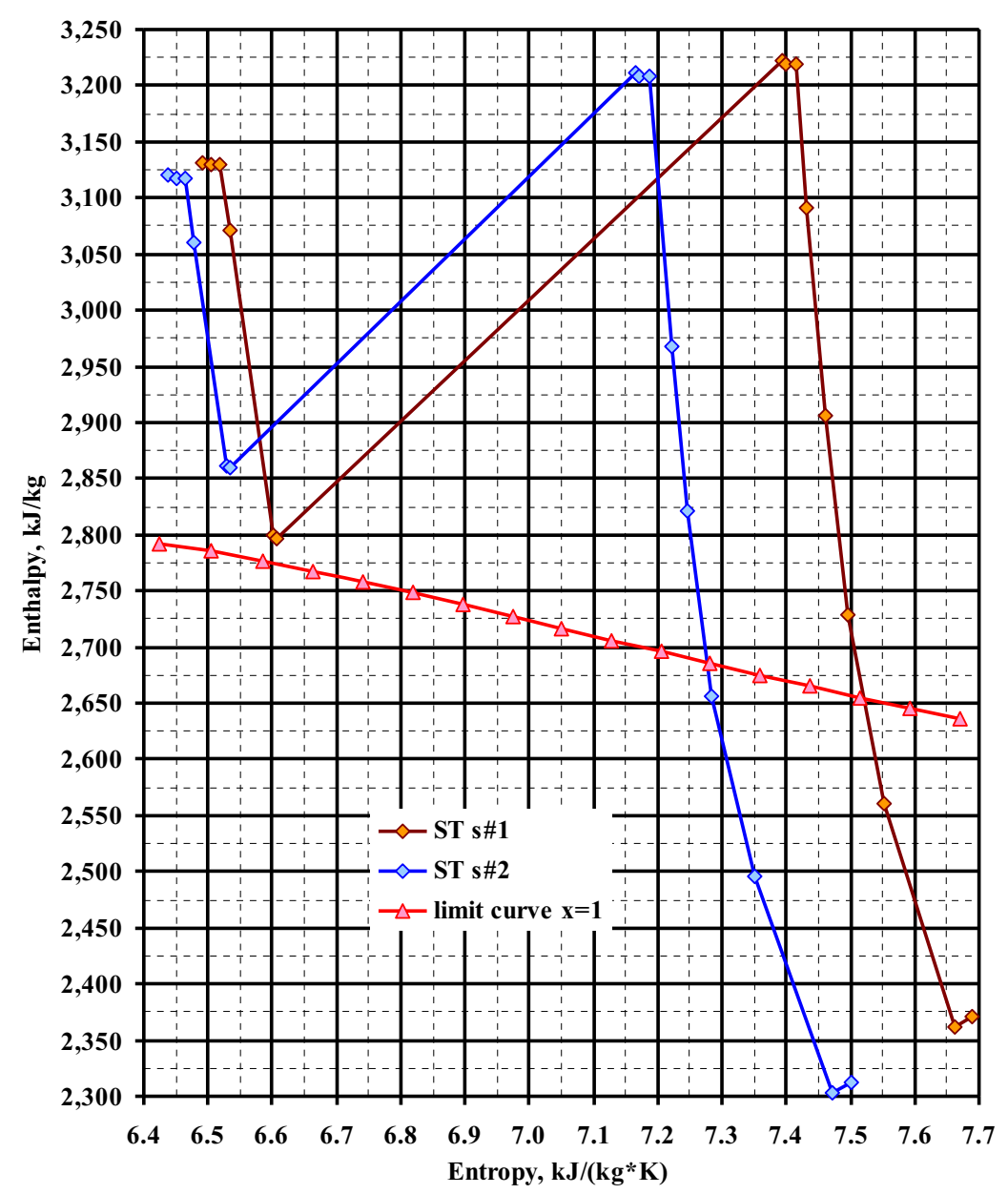

Fig. 5. The enthalpy-entropy diagram for the processes in the ST, in the two cases.

expansion process in ST for the two schemes. The energetic flow rates (columns $\Phi_{\text {en c } 1}$ and $\Phi_{\text {en c } 2}$ ) and the exergetic ones (columns $\Phi_{\text {ex c1 in }}, \Phi_{\text {ex c1 out }}, \Phi_{\text {ex c2 out }}$ ) are shown in Figure 6 . We used the following main notation: " $\Phi_{\mathrm{q}}$ ", for heat; "P" for mechanical work and power; " $\Delta$ ", for energy losses and exergy destructions; "1", for the first circuit; "2" for the second one. In this step, we obtained, also, practically the same values for:

- the exergetic efficiency of the SC: $\eta_{\text {ex SC s } \# 1}=80.974 \%$, $\eta_{\text {ex SC s } \# 2}=80.919 \%$;

- the power at generator clamps: $P_{\mathrm{gc} \mathrm{s} \# 1} \cong 36.136 \mathrm{MW}$, $P_{\mathrm{gc} \mathrm{s} \# 2} \cong 36.112 \mathrm{MW}$.

\section{Conclusions and future developments}

The paper presents a numerical modeling of the thermodynamic processes in SC TPP, with application on a CSPP-SC with medium temperatures to the hot heat source. The main contribution of the authors was achievement of a physical and mathematical model, for computing the thermodynamic processes into CSPP-SC in the reference regimes. The goal is to establish CSPP-SC performances, putting into evidence the energy losses and the exergy destructions. It required conceiving of dedicated functions and procedures and interconnecting them in our iterative software.

The main conclusion, determined by applying this model for diverse quantitative flows and qualitative parameters, is that, for the same SG exergetic efficiency could be obtained the same values of the global energetic efficiency, by using various pairs for: main steam pressure, and feeding water preheating temperature. In the previous presented case study, because the powers at generator clamps are practically the same, while the design with limited regenerative preheating requires fewer components and lower investment in "secondary" circuit, results that the $\mathrm{s} \# 2$ scheme is preferable from both points of view: technically and economically.

In a future work, we intend to do, into a similar approach, energetic and exergetic analyses on the steam cycle secondary circuit of solar power plants with heliostats and towers and thermal storage in the primary circuit with molten salts. 


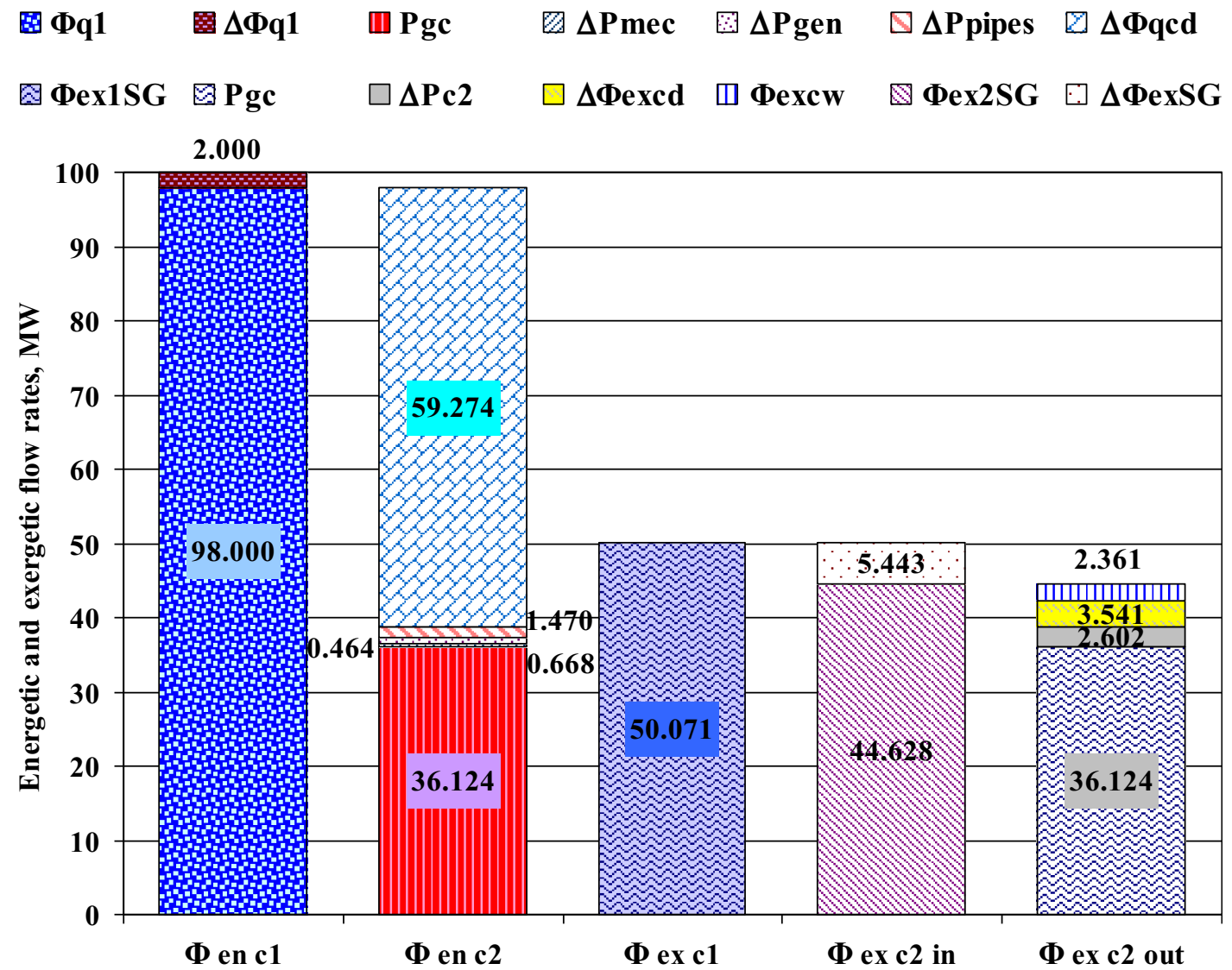

Fig. 6. The energetic and exergetic flows rates diagram for the analyzed CSPP-SC.

\section{References}

1. M.J. Emes, M. Arjomandi, G.J. Nathan, Effect of heliostat design wind speed on the levelised cost of electricity from concentrating solar thermal power tower plants, Sol. Energy 115, 441 (2015)

2. F.A. Al-Sulaiman, M. Atif, Performance comparison of different supercritical carbon dioxide Brayton cycles integrated with a solar power tower, Energy 82, 61 (2015)

3. A. Fernández-García, M.A. Cantos-Soto, M. Röger, C. Wieckert, C. Hutter, V. Martínez-Arcos, Durability of solar reflector materials for secondary concentrators used in CSP systems, Sol. Energy Mater. Sol. Cells 130, 51 (2014)

4. D.A. Baharoon, H.A. Rahman, W.Z.W. Omar, S.O. Fadhl, Historical development of concentrating solar power technologies to generate clean electricity efficiently - A review, Renew. Sustain. Energy Rev. 41, 996 (2015)
5. O. Behar, A. Khellaf, K. Mohammedi, S. Ait-Kaci, A review of integrated solar combined cycle system (ISCCS) with a parabolic trough technology, Renew. Sustain. Energy Rev. 39, 223 (2014)

6. J. Sun, Q. Liu, H. Hong, Numerical study of parabolic-trough direct steam generation loop in recirculation mode: characteristics, performance and general operation strategy, Energy Convers. Manage. 96, 287 (2015)

7. E.K. Burin, L. Buranello, P.L. Giudice, T. Vogel, K. Görner, E. Bazzo, Boosting power output of a sugarcane bagasse cogeneration plant using parabolic trough collectors in a feedwater heating scheme, Appl. Energy 154, 232 (2015)

8. H. Liang, S. You, H. Zhang, Comparison of different heat transfer models for parabolic trough solar collectors, Appl. Energy 148, 105 (2015)

Cite this article as: Victor-Eduard Cenuşă, George Darie, Diana Tuţică, Mihaela Norişor, Florin-Niculae Alexe, Chiriţă-Mihai Muşat, Energetic and exergetic analysis of Rankine cycles for solar power plants with parabolic trough and thermal storage, Renew. Energy Environ. Sustain. 1, 10 (2016) 